\title{
Confocal Laser Scanning Microscopy and Two Photon Excitation Microscopy as Tools to Study Testate Amoebae
}

\author{
Z. Burdíková ${ }^{* * *}$, M. Čapek ${ }^{* * * * *}$, P. Ostašov ${ }^{*}$, E.A.D. Mitchell ${ }^{* * * *}$, J. Machač $^{* * *}$, L. Kubínová \\ * Institute of Physiology, Academy of Sciences of the Czech Republic, v.v.i., Vídeňská 1083, Prague \\ 4 - Krč, 14220 Czech Republic \\ ** Institute of Geology and Palaeontology, Faculty of Science, Charles university, Albertov 6, \\ Prague, 12843 Czech Republic \\ *** Institute of Botany, Academy of Sciences of the Czech Republic, v.v.i., Lesní 322, Prühonice, \\ 25243 Czech Republic \\ **** Faculty of Biomedical Engineering, Czech Technical University in Prague, nám. Sítná 3105, \\ Kladno 2, 27201 Czech Republic \\ ***** WSL Swiss Federal Research Institute, Wetlands Research Group, Lausanne, Switzerland
}

Testate amoebae (TA) (Protozoa: Rhizopoda) are a group of unicellular animals $(20-400 \mu \mathrm{m})$ protected by a test. The test encloses the cell plasma and has usually a single aperture for the pseudopodia. A proteinaceous organic matrix is the basic test component. There are four main test types: proteinaceous (species with a flexible or rigid test), calcareous, siliceous (species which secrete their own regular siliceous test plates, so-called idiosomes) and agglutinated (species which include extraneous mineral particles in their test structure, so-called xenosomes).

TA are commonly used as model organisms in population ecology, ecotoxicology and paleoecology, for their cosmopolitan dispersion and species-specific ecological preferences together with low ecological valence to changes of environmental surroundings [1]. TA play a very important role in most aquatic and terrestrial ecosystems and are, therefore, an essential subject of many scientific studies, such as paleoecological, environmental, etc. [2]. However, only restricted number of articles was published about their morphology, biometry and ecological preferences [3]. TA classification is based on the detailed study of structure of the test. The cytoplasm, the types of pseudopodia and nuclei for the majority of these species have not been yet observed and their visualisation will help with systematic identification.

In many of TA-based ecological studies it is important to know precise estimates of test volumes. The volume of biomass inside the tests can then be calculated with the help of appropriate conversion factor. The volume of biomass is usually evaluated using simple measurements and using geometrical shapes of TA tests. This approach may not be accurate.

The most common technique used for studying TA is conventional wide-field optical microscopy, more specifically bright-field and phase contrast microscopy. However, data produced by this technique have low image contrast. Generally, wide-field methods are not powerful enough to distinguish important features of the test or the inner structures of the cell.

More convenient and widely used tool is electron microscopy: scanning electron microscopy (SEM) and environmental SEM (ESEM). By using these techniques it is possible to examine the surface features of TA with famously high resolution, reasonably good depth of field and to perform quantitative elemental analyses of shell structures. However, SEM and ESEM cannot be used to examine living TA or internal structures of the amoeba. Moreover, for species determination using 
morphological features, the test often needs to be examined from all sides and the interior structures need to be visible.

We tried to avoid above mentioned limitations by using microscopic techniques - confocal laser scanning microscopy (CLSM) and two photon excitation microscopy (TPEM) the use of which, according to our best knowledge, was not described in this field yet. These techniques nowadays offer high resolution and real-time cell imaging. The knowledge of the optical properties of the intracellular organelles obtained by their using is deemed to bring valuable information about cell morphology, cellular internal processes or organelles spatial distribution [4].

The main goal of the present study was to show the advantages of CLSM and TPEM to study TA. We demonstrate using CLSM, TPEM and various fluorescent dyes to visualize TA tests, inner structures of TA, their organelles, and symbionts. The acquired data and 3D visualizations [5] created from them enable us to study morphology of TA structures and measure precisely volume of inner biomass of the amoeba inside the test using stereological methods [6]. Imaging of TA morphology was applied to confirm taxonomic descriptions and for the structural and biochemical imaging of living specimen. Moreover, ecologists often face challenges with the identification of TA. We show that 3D visualization of CLSM and TPEM data is useful for this task as well.
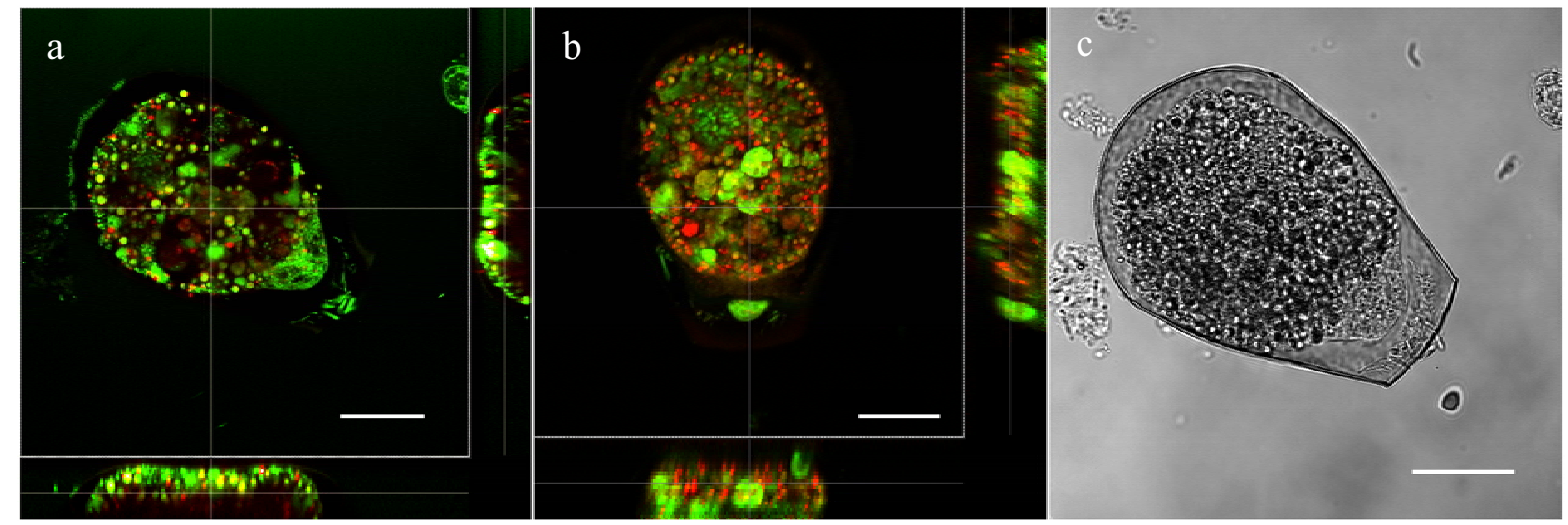

FIG. 1: Hyalosphenia papilio

1a) organic components (green, FITC), symbionts with chlorophyll a (red), $z=30 \mathrm{um}$, CLSM.

1b) organic components (green, FITC), symbionts with chlorophyll a (red), $z=50 \mathrm{um}$, TPEM.

1c) transmission channel of $1 \mathrm{a}$; the scalebar is $50 \mathrm{um}$.

\section{References}

[1] R.K Booth, J Paleolimnol. 28 (2002) 329-348.

[2] E.A.D. Mitchell et al., J Paleolimnol. 40 (2008) 603-618.

[3] M. Vohník, Microbial Ecology 57 (2009) 203-214.

[4] F. Charriere, Optics Express 14 (2006) 7005-7013.

[5] M. Čapek et al., Microsc. Res. \& Tech. 72 (2009) 110-119.

[6] L. Kubínová, A. Diaspro, Confocal and two photon microscopy: foundations, applications, and advances, Wiley-Liss Inc., New York, 2002

[7] This research was supported by the Ministry of Education, Youth and Sports of the Czech Republic under contracts No. MSM6840770012 and LC06063, and by the Czech Science Foundation under contracts No. 102/08/0691 and 304/09/0733. 